\title{
Design and Realization of Greenhouse Environment Information Analysis and Processing System
}

\author{
Xiaoying Sun \\ NanChang Institute of Science \& Technology,Nanchang,330108
}

Keyword: Vegetables; Information processing;Monitoring; Environment

\begin{abstract}
Most of the domestic greenhouse management lack of effective information technology means that the monitoring of the growth environment of greenhouse crops depends mainly on human experience. The precision and reliability need to be improved. At the same time, the successful planting experience is hard to be summarized and utilized. Automated detection and control to improve the environmental conditions in the greenhouse and intelligent management help to obtain high-quality crops and achieve high yields, thus obtaining considerable economic and social benefits. Therefore, it is of practical significance to study related technologies and develop environment information analysis and processing system. The system studied in this paper has improved the accuracy and response speed of the software and has made the early warning function well applied. On the hardware side, the production cost is reduced without affecting the quality. Although some areas still need to be improved, but it already have the value of application and promotion.
\end{abstract}

\section{Introduction}

With the progress of science and the advent of the information age, fresh blood has also been injected into the agricultural field. The backward production technology is gradually withdrawn from the stage of history. The maturity of Internet of Things makes it more widely used in various fields. The combination of Internet of Things technology and greenhouse technology has also diversified the technical means of facility agriculture and brought new ways of agricultural production.

Using wireless network technology transmission system for analysis can make the operator well aware of the situation inside the greenhouse, and based on the analysis to issue control command to the greenhouse, so as to adjust the greenhouse internal environment in order to achieve the best crop growth conditions, improve crop yield and quality. Vegetable production quality and safety control and related data platform have a profound impacton the development of the vegetable industry. For the optimization of industrial structure, improving economic efficiency, increasing farmers' income andperfecting the ecological environment is of great significance.

\section{Environmental Parameters and program design of the Overall System}

The quality of vegetable growth and development mainly depend on its own genetic factors and external growth environment. The greenhouse environmental information analysis and processing system built by this system mainly monitors and controls the growth conditions of cucumber and tomato. The environmental factors include temperature, humidity, $\mathrm{CO} 2$ concentration and light intensity.

Greenhouse Environmental Parameters. Different vegetables in the growth process have significant differencesof the temperature requirements, even to say the world is different. For the same type of vegetables, the temperature requirements are not the samein different period.

Humidity. Soil moisture can affect the nutrients that vegetables absorb from the soil. Too high humidity will cause the roots of vegetables is more shallow, resulting in wet damage, lack of oxygen and rotting roots. Too low will cause the vegetables to die because of wither and transpiration.

Carbon Dioxide Concentration. Carbon dioxide is an important part of photosynthesis of 
vegetables. Under the influence of sunshine, chloroplasts convert the carbon dioxideentering the leavesandwaterabsorbed by the rootsinto glucose and oxygen. When the concentration of carbon dioxide in the greenhouse reaches the appropriate standard for vegetable growth, the photosynthesis of vegetables is enhanced, the yield is significantly increased, and the quality is significantly improved.

Light Intensity. Some vegetables rely mainly on photosynthesis growth, so the light intensity for the growth of some vegetables is crucial. Inadequate illumination will lead to slow growth and developmentfor some plants, causing weak light, so that the weight of the fruit will be reduced, and the empty fruit androtten fruit increase.

\section{Greenhouse Environmental Information Analysis System Working Principle}

The greenhouse environmental information analysis and processing system consists of four parts: the sensor part is responsible for collecting parameters of the greenhouse control factors; the parameter analysis part is responsible for analyzing the collected parameters; the control and processing core part is responsible for issuing the control instructions according to the result; the executive part is responsible for executing the command. The whole execution process is to collect the parameters of the control factors in the greenhouse and then transmit them to the main control chip to compare the collected data with the preset values to obtain the analysis result and issue an order according to the result to control the execution of the corresponding actuator regulation order. These four parts form a closed-loop systemwith the greenhouse environment. Fig. 1.

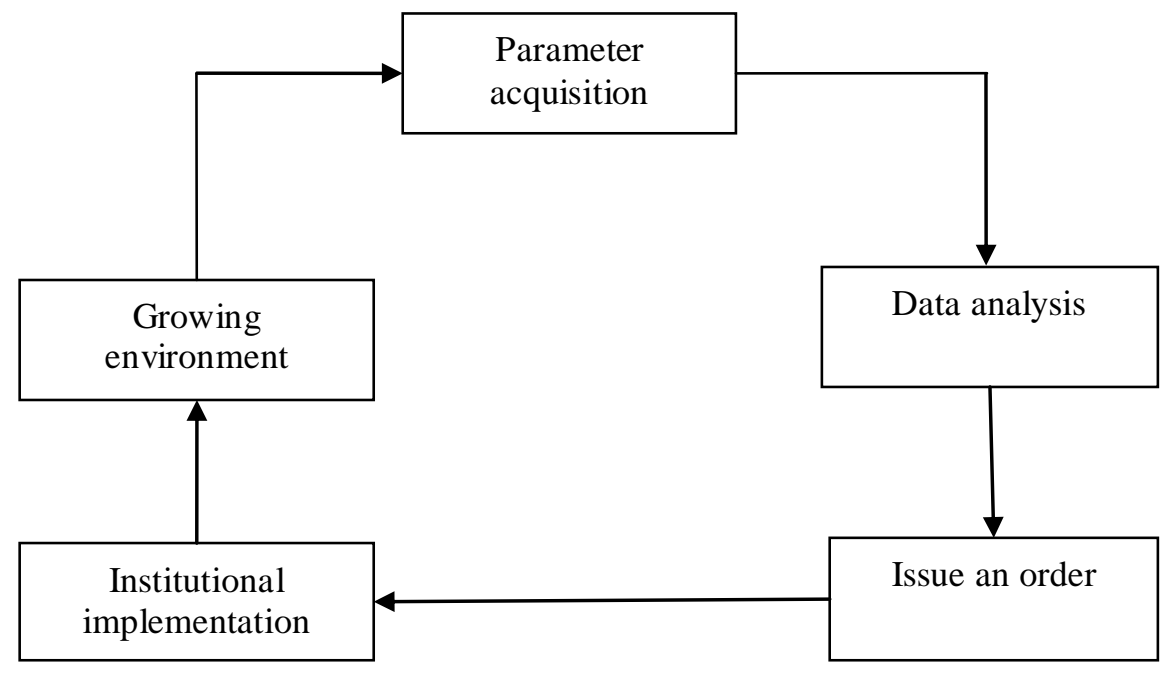

Figure 1. System Control Theory

\section{System Hardware Design}

Micro-control platformuses GTX TX-1C 51 microcontroller development board. It takes STC89C52RC microcontroller as the control core, peripheral components and expansion interfaces are rich. The microcontroller has a total of 40 pins, input and output I / O port P0, P1, P2, P3. Supply voltage $5 \mathrm{~V}$, reset function. The minimum system of the microcontroller is integrated, and the program download is convenient, fast execution, can meet the expectations of this article.

\section{System Software Design}

System control strategy is to focus on establishing a vegetable growth environment testing control system, in the cultivation of vegetables, to provide real-time environmental parameters, and carry out analysis and control, so as to cultivate more high-quality vegetables. In this system, each sensor will collect the environmental parameters of the data passed to the processor, after getting resolution, it will be sent to the host computerby wireless transmission, the host computer will 
receive and store the data and transmit it to the online client in real time, the received data can be detected in real time. According to the set threshold, it determines whether it is beyond the given range, and timely issue the alarm and make corresponding regulation.

In the main control flow of system, in the beginning, the first it completes the entire system initialization, define the first address of each register and the interrupt source initialization and so on. And then call the subroutine to collect data to detect and collect all the required factors in the environment and send it to the host computer through the wireless communication module to display the environmental parameters in real time from the host computer interface and then collect the data and system information to set the threshold ratio, and then decide whether it is necessary to issue control commands and SMS alarm; when it needs to issue control commands, through the wireless module to complete the host computer and the control chip communication, drive the implementing agencies to complete the adjustment of environmental parameters, and finally the cycle detection and control will start, real-time monitoring the environmental changes. Fig. 2 is the main control flow chart.

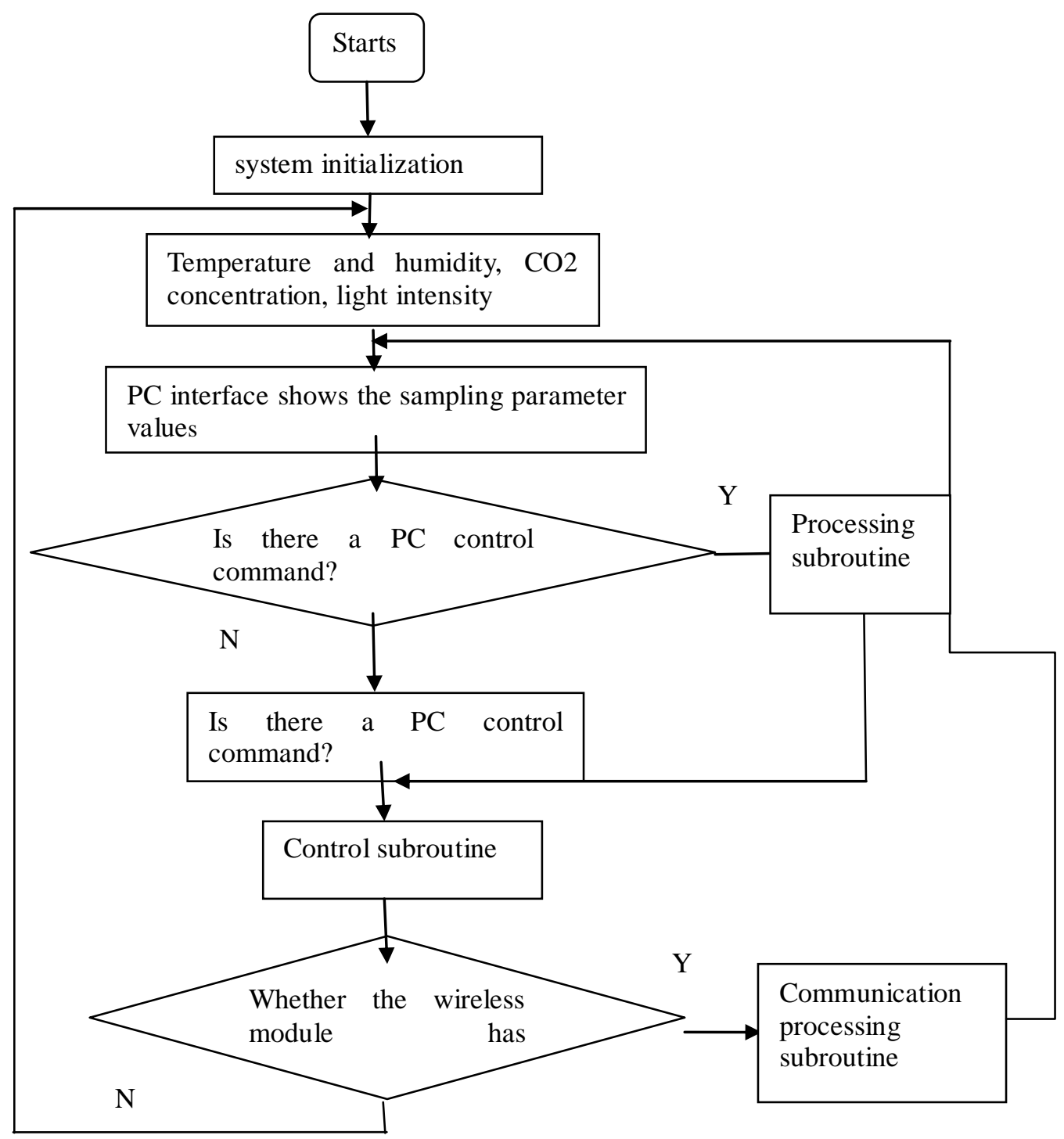

Figure 2. system main control flow chart 


\section{System Main Module and Function Introduction}

SMS Alert Module. SMS early warning system morecurrently is applied to the field of natural disasters such as weather forecasting early warning, rarely in other areas. The most used structure is Internet network terminal - Central database server -SMS platform.For some high-value cash crops, the impact of some minor environmental factors will breed a series of pests and diseases, thus bring a lot of economic losses. For each environmental parameter, it will be compared with the corresponding parameter threshold in the database. If it is higher or lower than the critical value, a corresponding SMS reminder is sent to the user belong to the hardware monitoring at this moment. The hardware control command message is forwarded to the remote hardware gateway according to the meaning of the message.

Greenhouse Status Information Inquiry Module.The main function of this module is based on a greenhouse as a basic unit, in the form of a graphical to displaythe user of the current greenhouse shed related information, when the system in the monitoring, once discover remote hardware sensors exceed the value, it will change its greenhouses icon; And with this module, when moving your mouse over the shed icon, it will show you all the relevant information about the shed. At the same time the system can automatically filter out a period of time you exceeded the greenhouse, it will be more beneficial to administrators have timely detected the problem shed and inform the user.

Mobile Client Communication Module.After the connection between the mobile client and the server is successfully established, firstly, the server returns the number of the shed owned by the user and the node information of each greenhouse as the string of the login user's mobile phone number. Once the server receives the signals from the remote hardware, by judging the hardware number, they will sent the message to the online users. Online users in accordance with the agreement can get the current greenhouse parameters and hardware status changes.

\section{Conclusion}

Vegetable is an important material that can not be separated from people's life. With the rapid development of the entire industry and the popularization of Internet application technology, the intelligent demand for vegetable greenhouses has also become higher. The use of Internet of Things to guide the cultivation of vegetables in greenhouse is an inevitable trend. Internet of Things is a new technology, which has been widely valued and applied by all countries in the world. It is characterized by comprehensive sensing, reliable transmission and intelligent processing, enabling the integration of information acquisition, processing and transmission. Relying on the Internet of Things technology to guide the cultivation of vegetables in greenhouses helps to digitize, refine and intelligently produce vegetables and thus help improve the quality of vegetables. The application of Internet of Things technology in vegetable production is an inevitable trend of development and can effectively solve the key problems in greenhouse vegetable production.

Fund project: Technology Project of Nanchang Institute of Science\& Technology(Project No. NGKJ-17-03)

\section{References}

[1] Oh S J. A Design of Intelligent Information System for Greenhouse Cultivation[J]. 2017, 15(2):183-190.

[2] Qiao Y, Zhang X, Cheng J. Environmental Information Control System for Small Greenhouse Based on ZigBee[J]. Electronic Science \& Technology, 2016.

[3] Nissimov S, Goldberger J, Alchanatis V. Obstacle detection in a greenhouse environment using the Kinect sensor[J]. Computers \& Electronics in Agriculture, 2015, 113(C):104-115.

[4] Mei Y J, Yan C, Zhang Z F, et al. Greenhouse environment regulation system based on ZigBee[J]. Electronic Design Engineering, 2017. 
[5] Fang J, Sun Z, Zhang X, et al. Development of MAV for Monitoring Spatial Distribution of Greenhouse Environment[J]. Journal of Agricultural Mechanization Research, 2016.

[6] Zhang X, Jia Y. A Wireless Multipoint Parameter Monitoring System for Greenhouse Environment[J]. 2016.

[7] Miao F, Wang Z, Tao B, et al. Greenhouse Environment Data Acquisition and Control System Design Based on ARM[J]. Journal of Agricultural Mechanization Research, 2015.

[8] Li S, Li M, Wang X. Design of greenhouse environment controller based on fuzzy adaptive algorithm[C]// Control and Decision Conference. IEEE, 2015:2644-2647.

[9] Pinchuk O. Apparatus and method for controlling a greenhouse environment[J]. 2016.

[10] Wang H, Hongli L I, Cheng Z, et al. Design of the Greenhouse Automatic Monitoring System Based on PLC[J]. Research \& Exploration in Laboratory, 2017.

[11] Spencer R. Late blight and greenhouse crops[J]. 2015.

[12]Tan F, Xue L J, Jiang S, et al. Remote Monitoring and Control System of Greenhouse Environment Based on Cloud Platform[J]. Journal of Mudanjiang Normal University, 2017. 